\title{
Ontogeny of solasodine-containing mucilage layer in Solanum viarum Dunal, ploidy types
}

\author{
MEENAKSHI SRINIVAS ${ }^{\dagger, s}$, Shyamsunder Joshi* and R KrishnaN ${ }^{\dagger}$ \\ Department of Botany, Bangalore University, Jnana Bharathi, Bangalore 560056, India \\ ${ }^{\dagger}$ Department of Ornamental Crops, Indian Institute of Horticultural Research, Hessaraghatta Lake PO, \\ Bangalore 560089 , India. \\ *Department of Botany, University of Agricultural Sciences, GKVK, Bangalore 560065 , India \\ Corresponding author (Fax, 091-080-8466291; Email, iihr@x400.nicgw.nic.in).
}

Berries of steroid-bearing Solanum viarum Dunal are exploited commercially in India as raw material by steroid industries for solasodine, a glycoalkaloid, present in the mucilaginous exotesta of the seed. Comparative ontogeny of exotesta studied through histochemical studies in diploid, autotetraploid and trisomic plants indicated similarity in the histochemical changes occurring during ontogeny of the outermost seed coat layer which culminated in the transformation of this layer into the mucilage layer. The increased cell size in this layer in the autotetraploid plants probably accounts for the higher steroid content reported. Corroborative evidences for histochemical changes observed in the mucilage layer were obtained from studies of ultrastructure using transmission electron microscopy.

\section{Introduction}

In Solanum viarum Dunal. (syn. S. khasianum var. chatterjeeanum), the mucilaginous coating around the seed (exotesta) has been reported to be very rich in glycoalkaloids, especially solasodine (Saini 1966; Yaniv et al 1981) which is used as raw material for the synthesis of steroid drugs in India. The induced autotetraploid in this species is reported to have higher content of solasodine in the berries (Krishnan 1983). Though the dynamics of accumulation and the role of roots in the synthesis of solasodine in berries of $S$. viarum have been investigated (Saini 1966; Meenakshi and Krishnan 1996; Nanaiah and Krishnan 1996) there are no reports on the ontogeny of the steroid-bearing structure. Hence, a study of the ontogeny of the layer was taken up through histochemical methods in diploid, autotetraploid and tertiary trisomic plants of $S$. viarum. Supportive ultrastructural studies using transmission electron microscopy were also carried out.

\section{Materials and methods}

\subsection{Histochemical studies}

The seeds from developing fruits of diploid, $\mathrm{C}_{6}$ generation autotetraploid (both healthy and aborted seeds) and tertiary trisomic plants were collected separately from plants grown in the field under uniform conditions. The material was collected regularly at an interval of 10 days (d) from the day of anthesis, until $80 \mathrm{~d}$ during which the fruits turned greenish yellow (70 d) and subsequently completely yellow $(80 \mathrm{~d})$. The seeds resulting from the above eight samplings were directly subjected to histochemical studies.

The seeds were fixed in two lots: in formalin-acetic acid-alcohol (1.1:18 by volume) to localize insoluble polysaccharides and Carnoy's B fixative (ethyl alcohol, chloroform and acetic acid in $6: 3: 1$ by volume) to localize nucleic acids and proteins. The material was dehydrated using ethanol-n-butanol grades and embedded in paraffin wax of $58^{\circ} \mathrm{C}$ melting point. Microtome sections of $\pm 8 \mu \mathrm{m}$ thickness were stained with periodic acidSchiff's (PAS) reagent for total insoluble polysaccharides, mercuric bromophenol blue for proteins and toluidine blue for nucleic acids before mounting in DPX medium.

In addition, at $60 \mathrm{~d}$ of development smears of the mucilaginous exotesta were stained with toluidine blue and iodine solution separately after processing in alcohol-nbutanol series following fixation in Carnoy's B fixative.

Keywords. Exotesta; histochemistry; mucilage; Solanum viarum; steroid; ultrastructure

J. Biosci., 23, No. 2, June 1998, pp 155-162. (C) Indian Academy of Sciences 


\subsection{Electron microscopy}

The seeds from the developing fruits of diploid, autotetraploid and tertiary trisomic were collected separately from plants grown in the field under uniform conditions. Diploid berries were sampled at 10,60,70 (greenish yellow) and 80 (yellow) days from the day of anthesis. The seed samples drawn at $10 \mathrm{~d}$ included placental tissues. In the other three stages, the mucilage layer around the seeds was separated and fixed. In tertiary trisomic and autotetraploid plants, berry samples were drawn at $70 \mathrm{~d}$ only. In autotetraploid plants, normal and aborted seeds were separately fixed based on visual differences in size and appearance.

The materials were fixed according to the procedure outlined by Karnovsky (1965), post-fixed in $1 \% \mathrm{OsO}_{4}$ in $0.1 \mathrm{M}$ phosphate buffer at $\mathrm{pH} 7.4$, dehydrated in alcohol with enblock staining in $2 \%$ uranyl acetate in $95 \%$ ethanol at $4^{\circ} \mathrm{C}$ for $1 \mathrm{~h}$. Ethanol was gradually replaced with acetone and then embedded in Epon araldite. The contrast of ultrathin sections was enhanced using uranyl acetate and lead citrate.

\section{Results}

The fruit of $S$. viarum is a many seeded globose berry. Seeds are compressed, discoid and small. The testa is crustaceous. The fruits of autotetraploid plants contained both fertile and aborted seeds. The outermost layer of the testa is made of thin-walled cells. The histochemical and ultrastructural changes noticed in the exotesta during the development of the seed in diploid, autotetraploid and tertiary trisomic plants are described here and the relevant micrometric observations at different intervals are presented in table 1.

There were no marked differences among the diploid, autotetraploid and tertiary trisomic plants in the histochemistry, ultrastructure and ontogeny of the exotesta, except for the differences in the thickness of the layer and size of nucleus and nucleolus in autotetraploids. Hence the following description holds good for all the three ploidy levels studied. However, there was no differentiation of the solasodine-accumulating layer in the aborted seeds of autotetraploids.
The sequence of events during the transformation of the outermost layer of the ovule into alkaloid bearing mucilage tissue can be divided into four phases. At 10 d after anthesis, the cells of the layer were compactly arranged with thin-walled cells. The tissue was metabolically very active and had dense cytoplasm rich in RNA and enlarged nuclei with densely stained nucleoli (figure 1A). There was no accumulation of polysaccharides. Proteins were also low. The ultrastructure of cells of this layer also indicated that the tissue was metabolically very active and was in an active phase of organelle proliferation and differentiation. The cytoplasmic organelles were found to occur at this stage in greater number than in other stages. The epidermal layer was covered by a thick cuticle. The large nuclei of the cells were proximally located and characterized by large nucleoli and electron-dense chromatin material (figure 4A). Mitochondria with prominent cristae were seen (figure 4B). Endoplasmic reticulum was heavily encrusted with ribosomes. The cytoplasm was rich in Golgi apparatus (figure 4B) and lysosomes besides large vacuole. Electron-dense circular particles of varying sizes were present in large vacuoles in cells of the outer layer which were absent in the immediately underlying layer of hypodermis.

For 30 to $60 \mathrm{~d}$ after anthesis, manifold increase in the size of the exotesta cells with a concomitant increase in their nuclear (figure 1B) and nucleolar diameters were recorded. Considerable accumulation of starch granules (figures 1C and 5A) and proteins was observed, but cytoplasmic RNA was low in concentration. Accumulation of reserve food material was evident from the presence of plastids with starch granules (figure 5B) in the highly vacuolated cytoplasm. With the onset of vacuolation, the cytoplasm, nucleus and other organelles were peripherally located. The large nucleus with prominent nucleolus contained dense chromatin material and a clearly defined nuclear wall. Smears of mucilagenous exotesta stained with toluidine blue and iodine solution separately indicated a larger nucleus and nucleolus in tetraploid than diploid and tertiary trisomic plants (figure $1 \mathrm{E}, \mathrm{F}$ ) as well as accumulation in all the three of starch grains around the nucleus (figure 1D).

Changes in the cells of the exotestal layer, at $70 \mathrm{~d}$

Table 1. Cytological values $(\mu \mathrm{m})$ for exotesta during development in diploid $(2 n)$, autotetraploid $(4 n)$ and tertiary trisomic $(2 n+1)$ plants in Solanum viarum ( \pm standard error).

\begin{tabular}{|c|c|c|c|c|c|c|c|c|c|}
\hline \multirow{2}{*}{$\begin{array}{l}\text { Days } \\
\text { after } \\
\text { anthesis }\end{array}$} & \multicolumn{3}{|c|}{ Exotesta thickness } & \multicolumn{3}{|c|}{ Nucleus diameter } & \multicolumn{3}{|c|}{ Nucleolus diameter } \\
\hline & $2 n$ & $4 n$ & $2 n+1$ & $2 n$ & $4 n$ & $2 n+1$ & $2 n$ & $4 n$ & $2 n$ \\
\hline 10 & $16.9 \pm 2.9$ & $18.3 \pm 2.0$ & $16.6 \pm 1.85$ & $11.7 \pm 1.0$ & $13.3 \pm 1.0$ & $11.9 \pm 0.95$ & $2 \cdot 1$ & $2.7 \pm 0.5$ & $2 \cdot 1$ \\
\hline 30 & $27.9 \pm 6.18$ & $30.4 \pm 4.93$ & $29.5 \pm 2.91$ & $12.9 \pm 0.83$ & $16.0 \pm 0.95$ & 12.5 & $2 \cdot 1$ & $3.6 \pm 0.52$ & $2 \cdot 1$ \\
\hline 50 & $36.6 \pm 6 \cdot 1$ & $40 \cdot 8 \pm 7 \cdot 15$ & $42 \cdot 0 \pm 5 \cdot 4$ & $13.3 \pm 1.0$ & $17.3 \pm 0.95$ & 12.6 & $2 \cdot 1$ & $3.9 \pm 0.47$ & $2 \cdot 1$ \\
\hline 60 & $139.0 \pm 18.6$ & $183.5 \pm 12.68$ & $153.5 \pm 11.9$ & $14 \cdot 1 \pm 1.9$ & $18.0 \pm 1.62$ & $14.9 \pm 1.49$ & $2 \cdot 1$ & $4 \cdot 2$ & $2 \cdot 3 \pm 0.28$ \\
\hline 70 & $172.6 \pm 24.9$ & $260.0 \pm 23.26$ & $176.4 \pm 18.9$ & $15 \cdot 4 \pm 2.7$ & $19.6 \pm 1.66$ & $15.8 \pm 4.07$ & $4 \cdot 2 \pm 2 \cdot 17$ & $5.4 \pm 1.9$ & $4.6 \pm 2.04$ \\
\hline 80 & $170.6 \pm 8.32$ & $235.0 \pm 18.72$ & $170.6 \pm 12.48$ & $14.6 \pm 2.08$ & $17.7 \pm 1.04$ & $14.6 \pm 2.08$ & $3.6 \pm 0.85$ & $4.8 \pm 0.95$ & $3.5 \pm 0.85$ \\
\hline
\end{tabular}



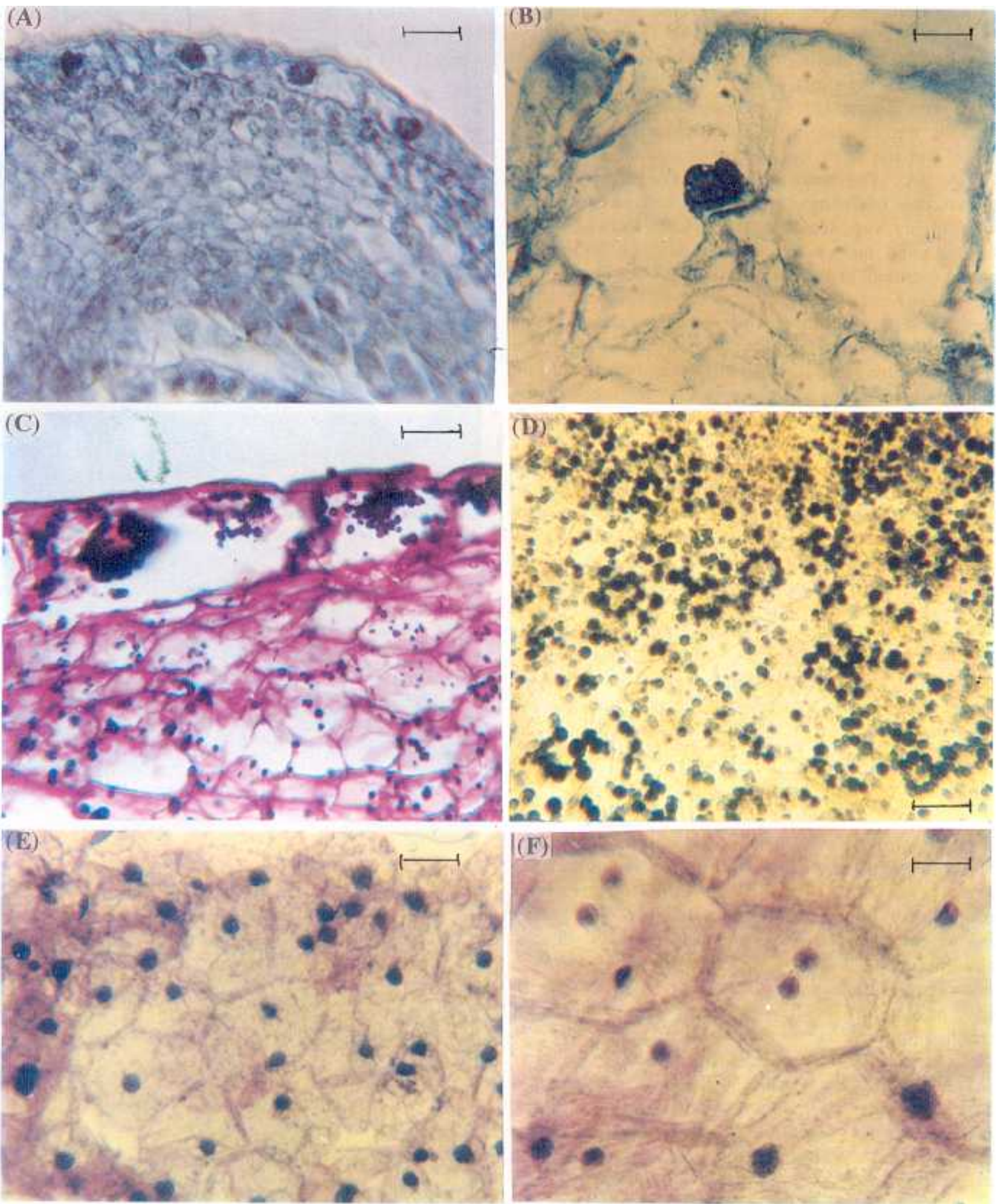

Figure 1. Exotestal layer at different stages of development. (A-D) diploid. (A) Densely stained nuclei with mercuric bromophenol blue at $10 \mathrm{~d}(\mathrm{Bar}=2.5 \mu \mathrm{m} ; \times 400)$. (B) Size increase in nucleus at $30 \mathrm{~d}(\mathrm{Bar}=2.0 \mu \mathrm{m} ; \times 500)$. (C) Starch grain accumulation around nucleus (PAS staining) at $50 \mathrm{~d}(\mathrm{Bar}=2.5 \mu \mathrm{m} ; \times 400)$. (D) Starch grain accumulation around nucleus in smear stained with iodine $(B a r=2.5 \mu \mathrm{m} ; \times 400)$. (E, F). Smears stained with toluidine blue at $50 \mathrm{~d}(\mathrm{Bar}=2.5 \mu \mathrm{m} ; \times 400)$. (E) Tertiary trisomic. (F) Autotetraploid, note larger nuclear size. 
after anthesis included: digestion of starch, synthesis of the glycoalkaloid and degeneration of the protoplast to a remnant. The cytoplasm stained intensely PAS-positive at this stage, but starch grains which were conspicuous in the previous stage of development were not seen. Proteins were absent in the cells of the exotesta at this stage. But the layer had intense concentration of RNA (figure 2A). The cross-sectional profile of cells under the electron microscope at this stage indicated that they were highly vacuolated with cytoplasm forming a thin layer around the vacuole and the nucleus occupied a corner position in the cell (figure 5B). Starch granules prominently seen in the plastids at the previous stage were not seen at this stage. Instead electron dense clumps could be seen (figure 5D).

At $80 \mathrm{~d}$ after anthesis, when the berries turn completely yellow, there was a slight decrease in thickness of the fully differentiated mucilaginous layer with a corresponding decrease in nuclear and nucleolar diameters. The accumulated PAS-positive substance showed signs of degradation at this stage. Ultrastructure of cells of the layer revealed that they were highly vacuolated with cytoplasm becoming so attenuated that the inner surface of plasma and tonoplast membranes were in contact at places. The electron-dense clumps which were prominent in the previous stage were not seen.

At $70 \mathrm{~d}$, in whole seed mounts, the exotestal layer can be distinguished as a white mucilaginous layer on the seed surface (figure 3 ). Lignification of the underlying hypodermal layer was observed at $70 \mathrm{~d}$ (figure $5 \mathrm{C}$ ) and $80 \mathrm{~d}$ (figure 2B).

\section{Discussion}

In $S$. viarum, the mucilaginous coating around the seed is reported to be very rich (25-27\%) in steroid, solasodine (Yaniv et al 1981). There are no earlier reports on the ontogeny of this steroid-bearing structure. The present study on the ontogeny of the layer in diploid, autotetraploid and tertiary trisomic plants was undertaken to compare histological or histochemical differences, if any, among them so as to identify the basis for the reported differences in solasodine content between diploids and autotetraploids (Krishnan 1983).

The present findings indicate that there are no significant differences among diploid, autotetraploid and tertiary trisomic plants with respect to the general ontogeny and histochemistry of the exotesta which acts as the solasodine-accumulating layer. During the development of the seed, the outermost layer of the seed (exotesta) undergoes significant histological and histochemical changes and ultimately transforms itself into a mucilaginous, solasodine-bearing structure. However, the tetraploid exhibited an increased thickness of the layer with a concomitant increase in the size of nuclei and

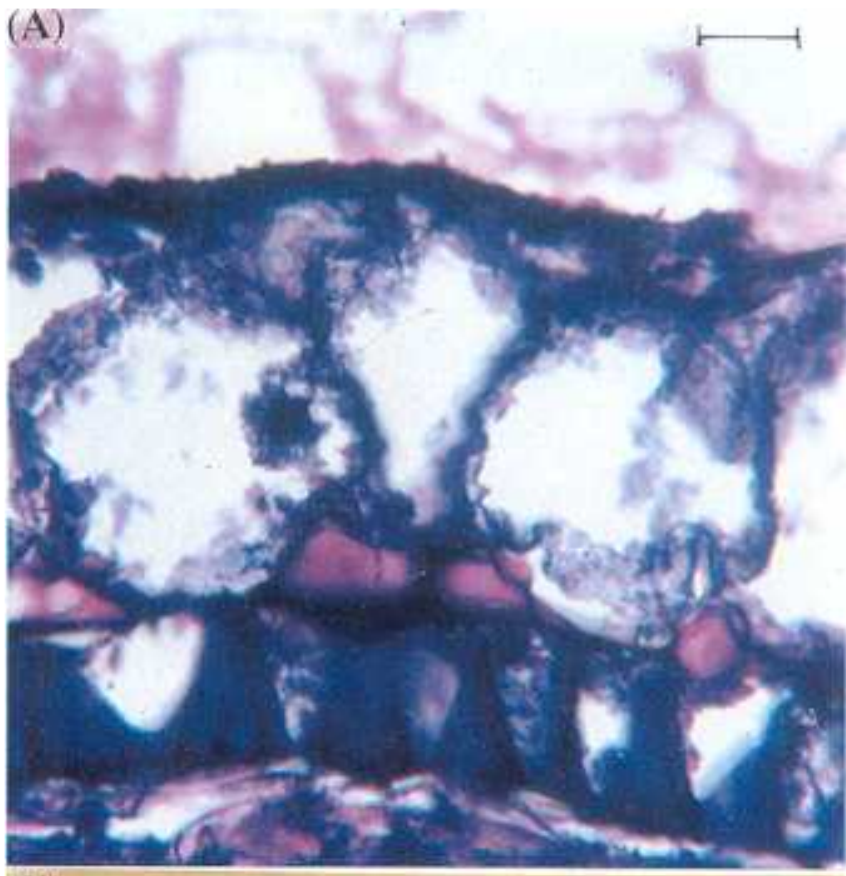

B)
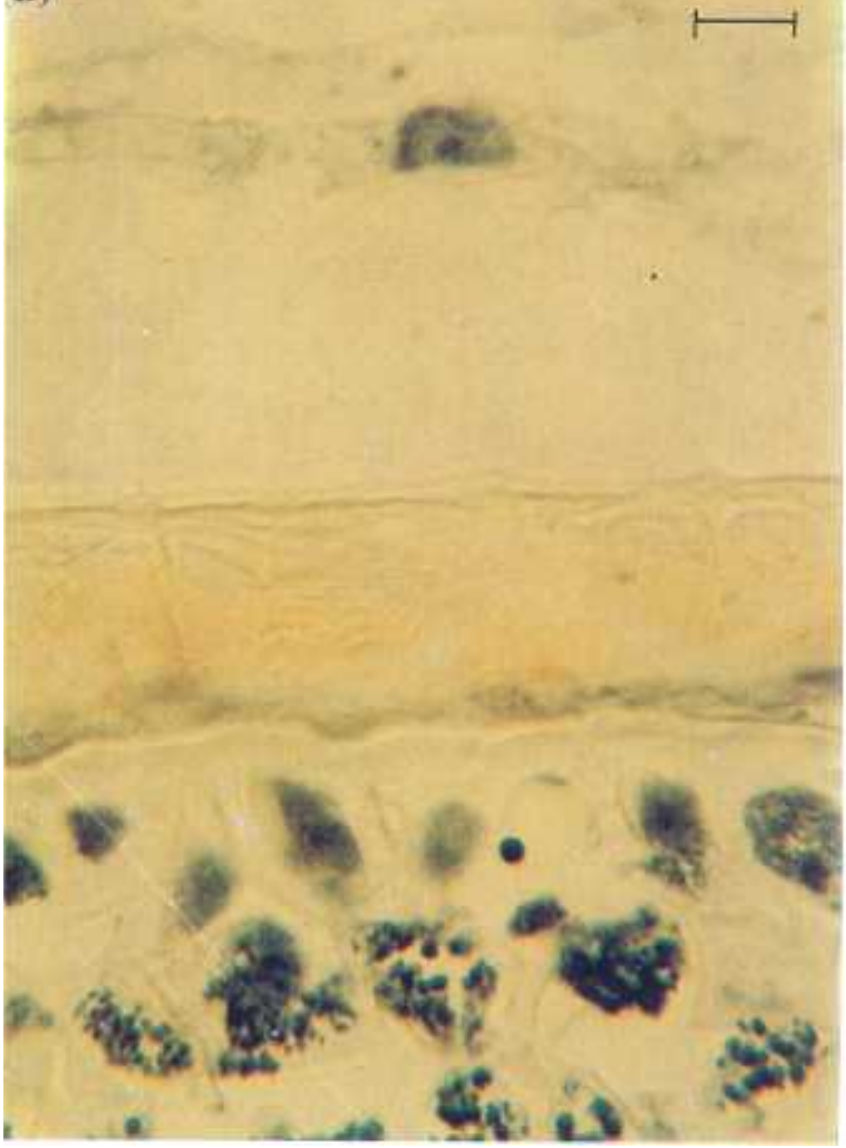

Figure 2. Exotestal and hypodermal layers in diploid. (A) RNA accumulation in exotestal layer and lignification of hypodermal layer (toluidine blue staining) at $.70 \mathrm{~d}(\mathrm{Bar}=2.5 \mu \mathrm{m}$; $\times 400$ ). (B) Degeneration of exotestal layer and lignified hypodermal layer at $80 \mathrm{~d}(\mathrm{Bar}=2.5 \mu \mathrm{m} ; \times 400)$. 
nucleoli which was observed as early as $10 \mathrm{~d}$ after anthesis. These variations may be attributed to the difference in ploidy level, as polyploids are known to exhibit gigasity. Solasodine content in berries of autotetraploids of $S$. viarum has been reported to be higher $(2.61 \%)$ than that of diploids [1.41-1.8\% dry weight basis (DWB); Krishnan 1983]. Based on the present findings, the difference in the solasodine content between the diploid and tetraploid plants might possibly be attributed to the increased thickness of the steroidbearing tissue in the autotetraploid.

Just after fruit set, i.e., $10 \mathrm{~d}$ after anthesis, the outer most layer of the seed comprised of a single layer of compactly arranged, thin-walled cells rich in cytoplasm, but devoid of polysaccharides and proteins. The cells of the layer were metabolically very active as indicated by the densely stained, enlarged nucleus and nucleolus as well as cytoplasm rich in RNA. The ultrastructure of the cells of this layer (in diploid) corroborates these observations. The cytoplasm was rich in rough endoplasmic reticulum, mitochondria, lysosomes and Golgi

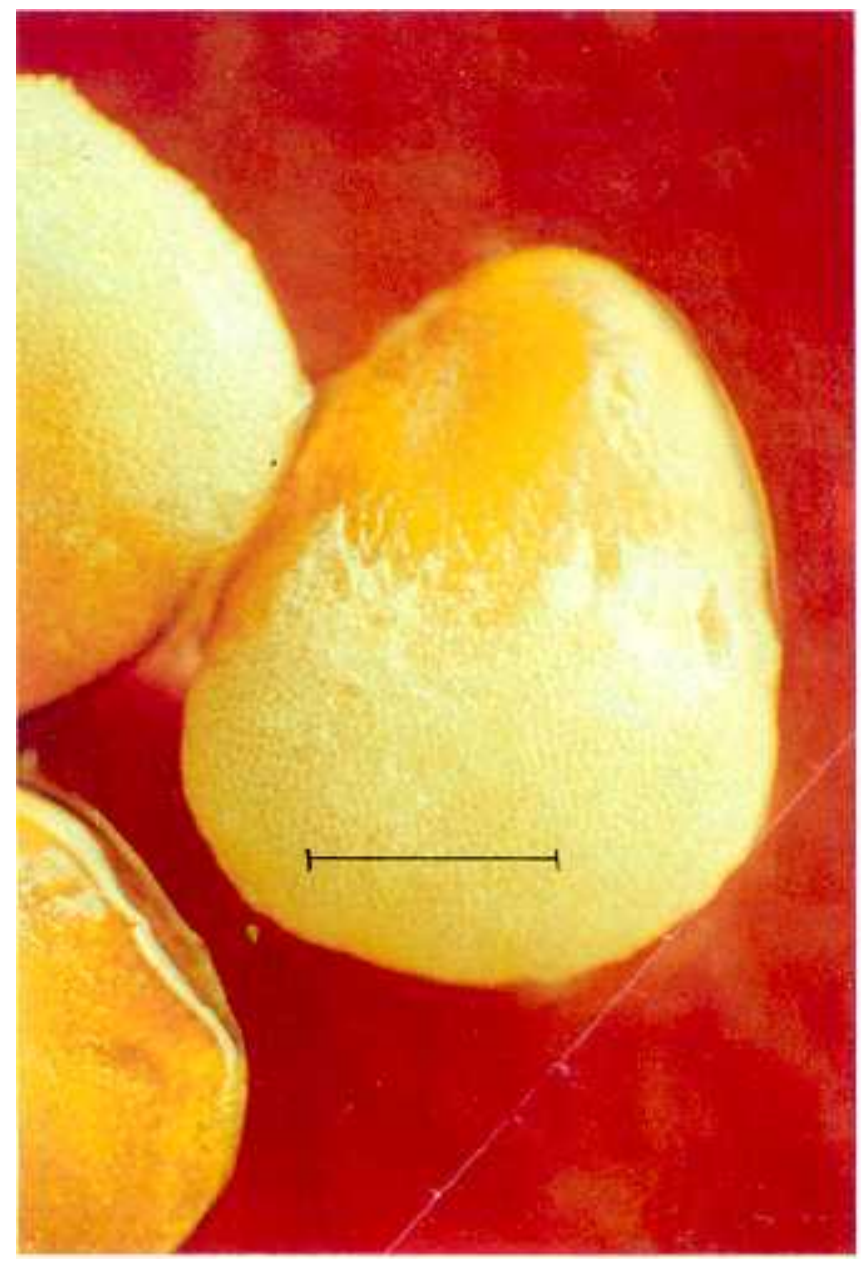

Figure 3. White mucilaginous coat on diploid seed $($ Bar $=1 \mathrm{~mm} ; \times 25)$ apparatus besides large vacuoles, which are indicative of high metabolic activity. It has been strongly suggested by James (1950) that alkaloids are synthesized principally in young, actively growing tissues, whose cells are either completely filled with cytoplasm or are rapidly vacuolating. He has also indicated that metabolically active tissues may be potential sites of alkaloid synthesis in whatever organ of the plant they occur.

With the progress in development of the seed, the cells of the exotesta of the seed continued to be metabolically active as indicated by a steady increase in the thickness of that layer with a corresponding increase in the size of nuclei and nucleoli. The cells of the layer continued to be devoid of proteins and polysaccharides, but rich in nucleic acids.

Further differentiation of the exotesta was observed at $60 \mathrm{~d}$ after anthesis, when there was a manifold increase in the size of cells of that layer. High accumulation of proteins and starch granules in the cells of exotesta was also observed at this stage. However, the cells exhibited low RNA content in the cytoplasm. Studies on the ultrastructure also revealed the presence of plastids with starch granules in the highly vacuolated cytoplasm.

The thickness of the layer increased significantly till $70 \mathrm{~d}$ after anthesis when the mature green berries just start turning yellow. Full differentiation of the alkaloid bearing structure was observed at this stage. Starch granules and proteins were conspicuously absent, but the cytoplasm was intensely PAS-positive, probably indicating that accumulated metabolites are fully utilized during the development of exotestal layer. The absence of starch granules was confirmed from studies on the ultrastructure of the mucilage layer at this stage. The presence of electron dense clumps was observed only at this stage.

A similar pattern of development and differentiation of mucilage-secreting epidermal cells of the seed coat has been reported in Plantago ovata by Hyde (1970). He found rapid cell expansion, increase in nuclear and nucleolar size and accumulation of starch, followed by deposition of mucilage inside the vacuoles. This was accompanied by shrinkage of cytoplasm and disappearance of starch. Fahn (1979) suggested that mucilage is produced in the epidermal cells of the seed coat which act as secretory tissues.

In referring to meristems as sites of alkaloid synthesis, James (1950) has postulated that alkaloid constituents may be built up in different parts of the plant to a relatively late stage of the assembly, but supplies for protein formation should reach the meristematic tissues in the form of sugars, amino acids and amides, since meristematic tissues are not primary importers of material into the plant. The final fitting together of these precursors and the synthesis of the alkaloid probably takes place in metabolically highly active tissues, like meristems, glandular tissues, cork cambia, etc. He has also suggested 

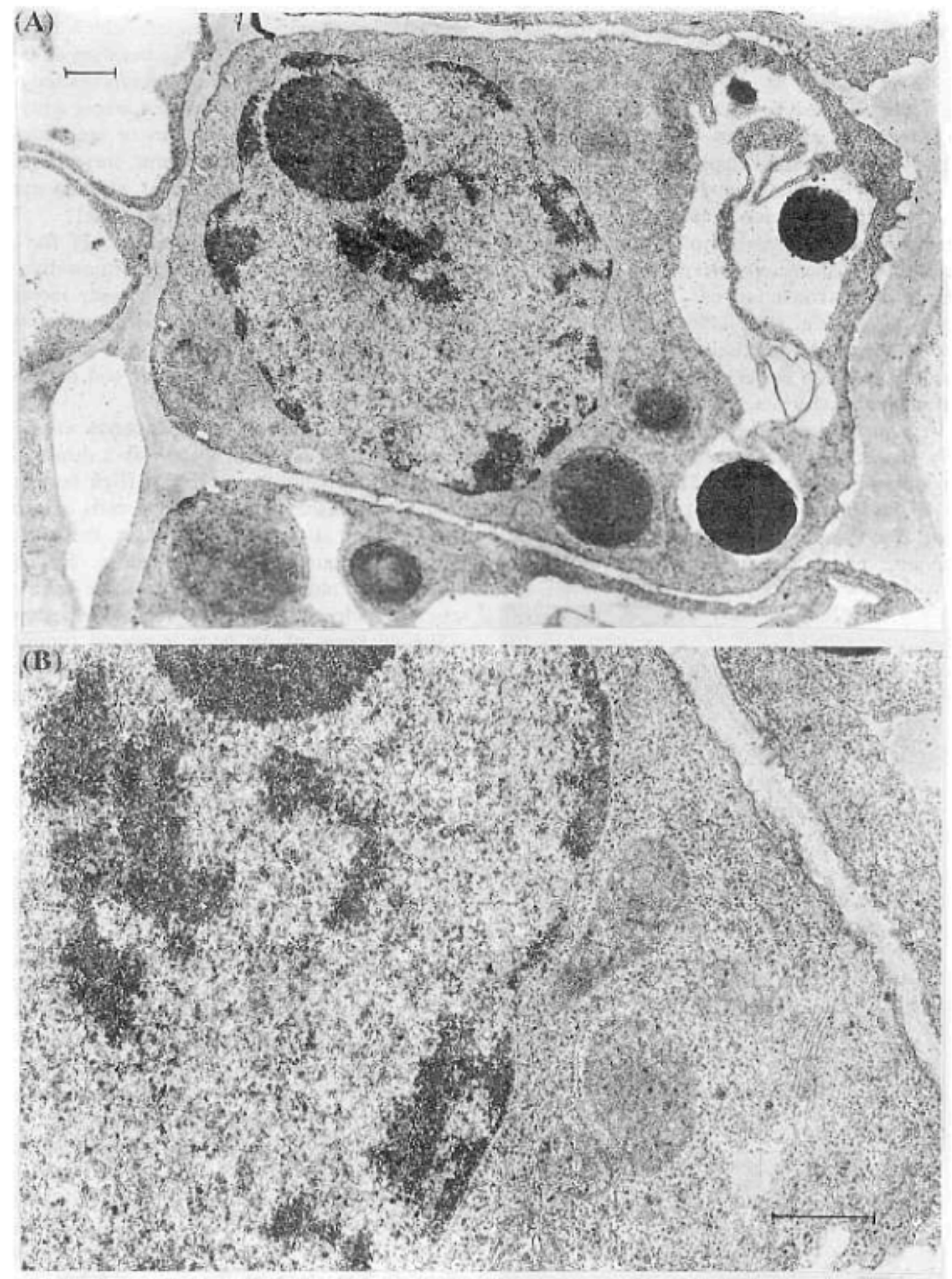

Figure 4. Exotestal layer at $10 \mathrm{~d}$ in díploid. (A) Conspicuous nucleus (N), nucleolus (nu) and mitochondria (M) $(B a r=1.0 \mu \mathrm{m} ; \times 8000)$. (B) Part of cell showing Golgi $(G C)$ and mitochondria $(M)$ in cytoplasm $(B a r=1.0 \mu \mathrm{m} ; \times 16000)$.

Figure 5. Exotestal layer of diploid. (A) Starch grains around nucleus at $50 \mathrm{~d}$ in semi-thin section $(1 \mu \mathrm{m})(\mathrm{Bar}=10 \mu \mathrm{m} ; \times 1200)$. (B) Starch grains (st) at $50 \mathrm{~d}$ in ultra-thin section (Bar $=5 \mu \mathrm{m} ; \times 5400$ ). (C) Differentiated mucilage layer with lignified hypodermal layer in semi-thin section at $70 \mathrm{~d}$ (Bar $=1.5 \mu \mathrm{m} ; \times 650$ ). (D) Nucleus (N) embedded in electron dense material (?steroid) at 70 $\mathrm{d}(\mathrm{Bar}=0.5 \mu \mathrm{m} ; \times 36000)$. 
Onto of mucilage layer in Solanum vianum

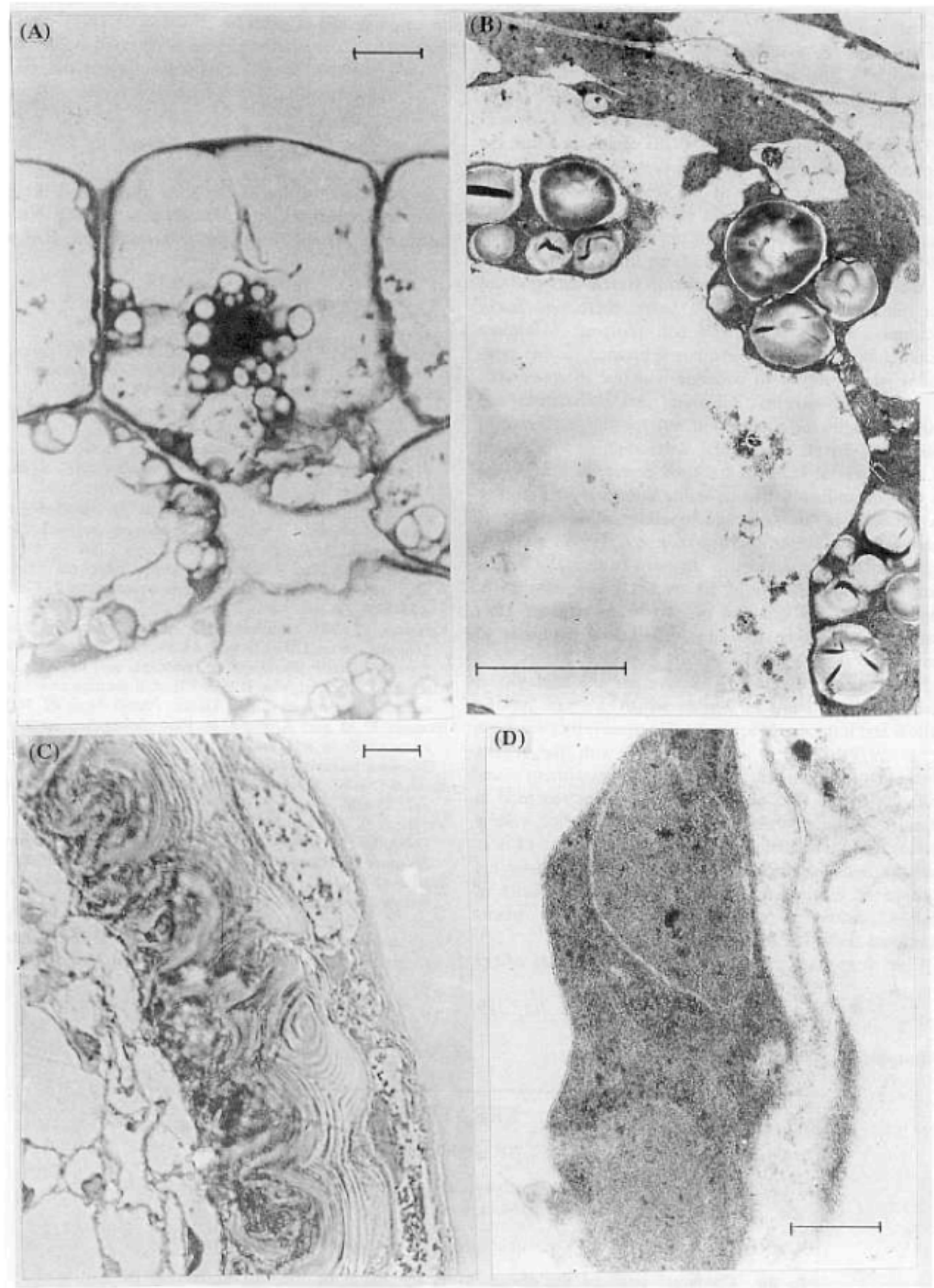


that alkaloids frequently arise through decomposition of the proteins, as evidenced by an increase in alkaloid content at a time when protein is breaking down.

Based on studies of the protein composition of lupine species by means of immunoelectrophoresis, Glowacki (1975 cf Waller and Nowacki 1978) established that the beginning of alkaloid production coincides with the disappearance of a majority of the storage proteins in the seed. The sequence of events in the present study in $S$. viarum are in conformity with the suggestions of James (1950) and Glowacki (1975). First the epidermal cells of the young seed were devoid of starch and proteins but rich in cytoplasmic RNA. Later, there was heavy accumulation of both starch and proteins. Maximum accumulation of a PAS-positive substance in the cytoplasm was observed to coincide with the disappearance of starch and proteins, indicating that both starch and proteins might have been utilized for the synthesis of solasodine. In all probability, solasodine is synthesized in this exotestal layer of the seed coat, which also acts as the accumulating tissue. While comparing solasodine biosynthesis in the seed and seedling callus of $S$. khasianum grown in vitro, Chaturvedi et al (1979) recorded higher solasodine content in the seed callus. The higher biosynthetic potentiality of the seed callus in vitro might conform with the situation in vivo supporting the view that seeds might be the sites of alkaloid synthesis in the plant.

It has been reported in $S$. viarum that solasodine content is maximum in berries which are just turning yellow and it decreases as the berries ripen fully (Varghese et al 1979). This is in conformity with the results obtained in the present study, wherein maximum accumulation of the PAS-positive substance was recorded in greenish yellow berries. But, in the fully ripe yellow berries, the accumulated substance showed signs of degradation and disappearance. This is corroborated by the absence of electron-dense clumps in exotestal cells of diploid, autotetraploid and tertiary trisomic plants examined under the electron microscope.

Thus, it appears that in $S$. viarum, the exotesta of the developing seed is probably the site of solasodine synthesis and accumulation. There is a rapid expansion of the cells of this layer during development culminating in its transformation into a mucilage layer.

\section{Acknowledgements}

The senior author thanks Dr Ram Mohan and his associates of Neuropathology Department of the National Institute of Mental Health and Neurosciences, Bangalore for their help.

\section{References}

Chaturvedi H C, Chowdhury A R and Uddin A 1979 Solasodine biosynthesis in seed and seedling-callus of $S$. khasianum Clarke grown in vitro; Indian J. Exp. Biol. 17 107-109

Fahn A 1979 Secretory tissues in plants (London: Academic Press) pp 751-759

Hyde B B 1970 Mucilage producing cells in the seed coat of Plantago ovata, developmental fine structure; Am. J. Bot. 57 1197-1206

James W O 1950 Alkaloids in the plant; in The alkaloids-chemistry and physiology (eds) R H F Manske and H L Holmes (New York: Academic Press) vol. 1, pp 1-87

Kamovsky M J 1965 A formaldehyde-glutaraldehyde fixative of high osmolarlity for use in electron microscopy; J. Cell Biol. A25 137-138

Krishnan R 1983 Multilocational varietal evaluation trial on Solanum viarum Dunal (syn. S. khasianum var. chatterjeanum); Proc. V. AICRP Workshop on medicinal and aromatic plants

Meenakshi S and Krishnan R 1996 Effect of grafting on solasodine content in Solanum viarum Dunal; Planta Med. 62 360-361

Nanaiah K M and Krishnan R 1996 Solasodine content and berry yieldş in self- and inter-ploidy grafts of steroid-bearing Solanum viarum; Indian Drugs 36 286-289

Saini A D 1966 Alkaloid content of S. khasianum Clarke; Curr. Sci. 23600

Varghese S, Sharma N S, Desai J D and Chiney J J 1979 Dynamics of solasodine biosynthesis and accumulation in Solanum khasianum Clarke; Indian J. Pharm. Sci. 41 198-200

Waller G R and Nowacki E K 1978 Alkaloid biology and metabolism in plants (London: Plenum Press)

Yaniv Z, Weissenberg M, Palevitch O and Levy A 1981 Effect of seed number and fruit weight on content and localization of glycoalkaloids in S. khasianum; Planta Med. 42 303-306

MS received 4 July 1997; accepted 16 March 1998

Corresponding editor: SIPRA GUHA-MUKHERJEE

\section{ERRATUM}

Journal of Biosciences, Vol. 23, No. 1, March 1998, pp 9-13.

Title: Interaction of indolicidin, a 13-residue peptide rich in tryptophan and proline and its analogues with model membranes.

Authors: C Subbalakshmi, V Krishnakumari, $N$ Sitaram and $R$ Nagaraj

In the 'Contents' page of the above issue, the name of the last author of the above paper was wrongly printed as $\mathrm{N}$ Nagaraj. It should be read as R Nagaraj. 